\title{
Decomposition Kinetics and Self-Preservation of Methane Hydrate Particles in Crude Oil Dispersions: Experiments and Theory
}

Artem A. Sizikov a, Valeriy A. Vlasov b, Andrey S. Stoporev a,c,d, Andrey Yu. Manakov a,c,"

a Nikolaev Institute of Inorganic Chemistry SB RAS, Ac. Lavrentievave. 3, Novosibirsk, 630090, Russian Federation, manakov@niic.nsc.ru

${ }^{b}$ Institute of the Earth Cryosphere, Tyumen Scientific Center, Siberian Branch of the Russian Academy of Sciences, P.O. Box 1230, 625000 Tyumen, Russian Federation

${ }^{c}$ Novosibirsk State University, Pirogova Str. 2, Novosibirsk, 630090, Russian Federation

${ }^{d}$ Gubkin University, Department of Physical and Colloid Chemistry, Leninsky prosp. 65, Building 1, Moscow, 119991, Russian Federation

* Corresponding author: Andrey Yu. Manakov, Dr.Sci., Head of laboratory, Nikolaev Institute of Inorganic Chemistry SB RAS, Ac. Lavrentiev ave. 3, Novosibirsk, 630090, Russian Federation, Tel. 7 (383)-316-53-46, Fax: +7 (383)-330-94-89. E-mail: manakov@niic.nsc.ru 
Table S1. Numerical data on the amounts of hydrate formed and decomposed in different experiments and at different stages of the experiments.

Experiment

(for more details see

$$
T_{\exp }{ }^{1},{ }^{\circ} \mathrm{C}
$$

Formation

Decomposition

Table 2 of the article)

\begin{tabular}{|c|c|c|c|c|c|}
\hline & & $N_{\text {formed }}{ }^{2}$, mol & $N_{\text {stage } 5}{ }^{5}, \mathrm{~mol}$ & $N_{\text {selfpres }}{ }^{6}, \mathrm{~mol}\left(\right.$ up to $\left.1^{\circ} \mathrm{C}\right)$ & $N_{1^{\circ} \mathrm{C}-20^{\circ} \mathrm{C}^{7}, \mathrm{~mol}}$ \\
\hline $\mathrm{O} 2-1$ & -4.8 & $0.0452^{3}$ & 0.0334 & 0.0008 & +0.0014 \\
\hline $\mathrm{O} 2-2$ & -10.2 & $0.0559^{3}$ & 0.0309 & 0.0004 & +0.0007 \\
\hline $\mathrm{O} 2-3$ & -13.6 & $0.0337^{3}$ & 0.0281 & 0.0059 & +0.0007 \\
\hline $\mathrm{O} 2-4$ & -18.8 & $0.0358^{3}$ & 0.0353 & 0.0010 & +0.0006 \\
\hline $\mathrm{O} 2-5$ & -10.8 & not measured & 0.0334 & 0 & -0.0009 \\
\hline O1-6 & -4.6 & $0.0578^{4}$ & 0.0526 & 0.0040 & +0.0008 \\
\hline O1-7 & -9.4 & $0.0562^{4}$ & 0.0435 & 0.0212 & +0.0007 \\
\hline O1-8 & -13.8 & $0.0593^{4}$ & 0.0463 & 0.0208 & +0.0007 \\
\hline O1-9 & -17.5 & $0.0597^{4}$ & 0.0367 & 0.0256 & +0.0011 \\
\hline O1-10 & -5.0 & $0.0269^{3}$ & 0.0211 & 0.0066 & -0.0010 \\
\hline O1-11 & -8.8 & $0.0292^{3}$ & 0.0166 & 0.0133 & -0.0010 \\
\hline
\end{tabular}

${ }^{1} T_{\text {exp }}$ - temperature of the experiment.

${ }^{2} N_{\text {formed }}$ amount of methane hydrate $\left(\mathrm{CH}_{4} \cdot 6 \mathrm{H}_{2} \mathrm{O}\right)$ formed at the stages $1-4$ of the experiments.

${ }^{3}$ Maximal amount of methane hydrate which can form in these experiments is 0.0370 mol that corresponds to 4 grams of water

${ }^{4}$ Maximal amount of methane hydrate which can form in these experiments is 0.0740 mol that corresponds to 8 grams of water

${ }^{5} N_{\text {stage } 5}$ - amount of methane hydrate $\left(\mathrm{CH}_{4} \cdot 6 \mathrm{H}_{2} \mathrm{O}\right)$ decomposed at the stage 5 of the experiments.

${ }^{6} N_{\text {selfpres }}$ - amount of methane hydrate $\left(\mathrm{CH}_{4} \cdot 6 \mathrm{H}_{2} \mathrm{O}\right)$ decomposed at the stage 6 of the experiments at temperatures up to $1^{\circ} \mathrm{C}$

(amount of hydrate which underwent self-preservation).

${ }^{7} N_{1{ }^{\circ} \mathrm{C}-20^{\circ} \mathrm{C}}$ - amount of methane emitted or consumed at the stage 6 of the experiments at temperatures above $1{ }^{\circ} \mathrm{C}$. 


\begin{tabular}{|c|c|c|c|c|c|c|c|c|c|c|c|c|c|c|c|}
\hline \multirow[t]{2}{*}{ Exp.* } & $\begin{array}{c}T_{\exp }, \\
{ }^{\circ} \mathrm{C}\end{array}$ & \multicolumn{14}{|c|}{$\alpha \mathrm{t}$ - transformation degree of hydrate to ice at time $\mathrm{t}, \mathrm{min} ; r_{\mathrm{d}}-$ decomposition rate, $\operatorname{mol} \min ^{-1}$ at time $\mathrm{t}, \mathrm{min}$} \\
\hline & & $\alpha 0$ & $\begin{array}{c}r_{\mathrm{d}} \\
t=0\end{array}$ & $\alpha 4$ & $\begin{array}{c}r_{\mathrm{d}} \\
t=4\end{array}$ & $\alpha 7$ & $\begin{array}{c}r_{\mathrm{d}} \\
t=7\end{array}$ & $\alpha 10$ & $\begin{array}{l}r_{\mathrm{d}} \\
t=10\end{array}$ & $\alpha 20$ & $\begin{array}{l}r_{\mathrm{d}} \\
t=20\end{array}$ & $\alpha 30$ & $\begin{array}{l}r_{\mathrm{d}} \\
t=30\end{array}$ & $\alpha 40$ & $\begin{array}{l}r_{\mathrm{d}} \\
t=40\end{array}$ \\
\hline $\mathrm{O} 2-1$ & -4.8 & 0 & 0.01090 & 0.714 & $12.94 \cdot 10^{-4}$ & 0.798 & $7.26 \cdot 10^{-4}$ & 0.848 & $5.04 \cdot 10^{-4}$ & 0.936 & $1.36 \cdot 10^{-4}$ & 0.959 & - & 0.956 & - \\
\hline $\mathrm{O} 2-2$ & -10.2 & 0 & 0.00428 & 0.406 & $14.07 \cdot 10^{-4}$ & 0.505 & $6.70 \cdot 10^{-4}$ & 0.556 & $4.50 \cdot 10^{-4}$ & 0.649 & $2.16 \cdot 10^{-4}$ & 0.700 & $1.36 \cdot 10^{-4}$ & 0.738 & $9.83 \cdot 10^{-5}$ \\
\hline $\mathrm{O} 2-3$ & -13.6 & 0 & 0.00229 & 0.263 & $8.86 \cdot 10^{-4}$ & 0.327 & $5.71 \cdot 10^{-4}$ & 0.368 & $4.40 \cdot 10^{-4}$ & 0.462 & $2.30 \cdot 10^{-4}$ & 0.518 & $1.50 \cdot 10^{-4}$ & 0.541 & $1.10 \cdot 10^{-4}$ \\
\hline $\mathrm{O} 2-4$ & -18.8 & 0 & 0.00187 & 0.202 & $14.03 \cdot 10^{-4}$ & 0.303 & $10.80 \cdot 10^{-4}$ & 0.383 & $8.54 \cdot 10^{-4}$ & 0.570 & $5.24 \cdot 10^{-4}$ & 0.689 & $3.34 \cdot 10^{-4}$ & 0.769 & $2.39 \cdot 10^{-4}$ \\
\hline $\mathrm{O} 2-5$ & -10.8 & 0 & 0.00304 & 0.308 & $19.18 \cdot 10^{-4}$ & 0.458 & $14.74 \cdot 10^{-4}$ & 0.578 & $11.21 \cdot 10^{-4}$ & 0.802 & $4.49 \cdot 10^{-4}$ & 0.889 & $1.72 \cdot 10^{-4}$ & 0.928 & $8.8 \cdot 10^{-5}$ \\
\hline $01-6$ & -4.6 & 0 & 0.00270 & 0.185 & $11.29 \cdot 10^{-4}$ & 0.238 & $7.74 \cdot 10^{-4}$ & 0.275 & $6.32 \cdot 10^{-4}$ & 0.362 & $3.87 \cdot 10^{-4}$ & 0.422 & $2.89 \cdot 10^{-4}$ & 0.466 & $2.29 \cdot 10^{-4}$ \\
\hline O1-7 & -9.4 & 0 & 0.00332 & 0.199 & $8.93 \cdot 10^{-4}$ & 0.235 & $6.10 \cdot 10^{-4}$ & 0.260 & $4.75 \cdot 10^{-4}$ & 0.317 & $2.95 \cdot 10^{-4}$ & 0.356 & $2.12 \cdot 10^{-4}$ & 0.386 & $1.71 \cdot 10^{-4}$ \\
\hline $01-8$ & -13.8 & 0 & 0.00217 & 0.125 & $14.41 \cdot 10^{-4}$ & 0.173 & $6.86 \cdot 10^{-4}$ & 0.198 & $4.61 \cdot 10^{-4}$ & 0.247 & $2.56 \cdot 10^{-4}$ & 0.279 & $1.75 \cdot 10^{-4}$ & 0.303 & $1.41 \cdot 10^{-4}$ \\
\hline O1-9 & -17.5 & 0 & 0.00027 & 0.017 & $3.88 \cdot 10^{-4}$ & 0.038 & $5.37 \cdot 10^{-4}$ & 0.064 & $5.91 \cdot 10^{-4}$ & 0.155 & $4.65 \cdot 10^{-4}$ & 0.205 & $2.34 \cdot 10^{-4}$ & 0.236 & $1.58 \cdot 10^{-4}$ \\
\hline O1-10 & -5.0 & 0 & 0.00102 & 0.145 & $5.56 \cdot 10^{-4}$ & 0.187 & $3.04 \cdot 10^{-4}$ & 0.216 & $2.23 \cdot 10^{-4}$ & 0.277 & $1.22 \cdot 10^{-4}$ & 0.317 & $9.5 \cdot 10^{-5}$ & 0.347 & $6.8 \cdot 10^{-5}$ \\
\hline \multirow[t]{2}{*}{ O1-11 } & -8.8 & 0 & 0.00105 & 0.137 & $6.73 \cdot 10^{-4}$ & 0.185 & $3.46 \cdot 10^{-4}$ & 0.213 & $2.39 \cdot 10^{-4}$ & 0.267 & $1.23 \cdot 10^{-4}$ & 0.300 & $8.4 \cdot 10^{-5}$ & 0.325 & $5.4 \cdot 10^{-5}$ \\
\hline & & $\alpha 50$ & $\begin{array}{l}r_{\mathrm{d}} \\
t=50\end{array}$ & $\alpha 60$ & $\begin{array}{c}r_{\mathrm{d}} \\
t=60\end{array}$ & $\alpha 120$ & $\begin{array}{c}r_{\mathrm{d}} \\
t=120\end{array}$ & $\alpha 180$ & $\begin{array}{c}r_{\mathrm{d}} \\
t=180\end{array}$ & $\alpha 240$ & $\begin{array}{c}r_{\mathrm{d}} \\
t=240\end{array}$ & $\alpha 300$ & $\begin{array}{c}r_{\mathrm{d}} \\
t=300\end{array}$ & $\alpha 360$ & $\begin{array}{c}r_{\mathrm{d}} \\
t=360\end{array}$ \\
\hline $\mathrm{O} 2-1$ & -4.8 & 0.968 & $2.5 \cdot 10^{-5}$ & 0.971 & $1.9 \cdot 10^{-5}$ & 0.975 & $4.8 \cdot 10^{-6}$ & 0.976 & $3.0 \cdot 10^{-6}$ & 0.978 & - & - & - & - & - \\
\hline $\mathrm{O} 2-2$ & -10.2 & 0.767 & $9.27 \cdot 10^{-5}$ & 0.797 & $8.3 \cdot 10^{-5}$ & 0.908 & $4.9 \cdot 10^{-5}$ & 0.958 & $1.9 \cdot 10^{-5}$ & 0.976 & $1.4 \cdot 10^{-5}$ & 0.983 & $0.94 \cdot 10^{-5}$ & 0.985 & - \\
\hline $\mathrm{O} 2-3$ & -13.6 & 0.582 & $8.32 \cdot 10^{-5}$ & 0.606 & $7.2 \cdot 10^{-5}$ & 0.687 & $3.2 \cdot 10^{-5}$ & 0.734 & $2.0 \cdot 10^{-5}$ & 0.768 & $1.5 \cdot 10^{-5}$ & 0.797 & $1.8 \cdot 10^{-5}$ & 0.821 & $1.2 \cdot 10^{-5}$ \\
\hline $\mathrm{O} 2-4$ & -18.8 & 0.827 & $1.66 \cdot 10^{-4}$ & 0.865 & $7.3 \cdot 10^{-5}$ & 0.949 & $1.4 \cdot 10^{-5}$ & 0.963 & $0.51 \cdot 10^{-5}$ & 0.968 & $0.14 \cdot 10^{-5}$ & 0.971 & $0.14 \cdot 10^{-5}$ & - & - \\
\hline $\mathrm{O} 2-5$ & -10.8 & 0.952 & $4.3 \cdot 10^{-5}$ & 0.964 & $3.0 \cdot 10^{-5}$ & 0.990 & $0.47 \cdot 10^{-5}$ & 0.998 & - & - & - & - & - & - & - \\
\hline $01-6$ & -4.6 & 0.501 & $1.85 \cdot 10^{-4}$ & 0.532 & $15.3 \cdot 10^{-5}$ & 0.644 & $8.2 \cdot 10^{-5}$ & 0.712 & $5.0 \cdot 10^{-5}$ & 0.760 & $4.1 \cdot 10^{-5}$ & 0.795 & $2.8 \cdot 10^{-5}$ & 0.820 & $2.2 \cdot 10^{-5}$ \\
\hline O1-7 & -9.4 & 0.411 & $1.44 \cdot 10^{-4}$ & 0.432 & $12.6 \cdot 10^{-5}$ & 0.518 & $6.9 \cdot 10^{-5}$ & 0.573 & $4.4 \cdot 10^{-5}$ & 0.613 & $3.4 \cdot 10^{-5}$ & 0.642 & $2.7 \cdot 10^{-5}$ & 0.666 & $2.0 \cdot 10^{-5}$ \\
\hline O1-8 & -13.8 & 0.322 & $1.16 \cdot 10^{-4}$ & 0.338 & $10.2 \cdot 10^{-5}$ & 0.404 & $5.8 \cdot 10^{-5}$ & 0.448 & $4.2 \cdot 10^{-5}$ & 0.480 & $3.4 \cdot 10^{-5}$ & 0.507 & $2.7 \cdot 10^{-5}$ & 0.529 & $2.3 \cdot 10^{-5}$ \\
\hline O1-9 & -17.5 & 0.257 & $1.18 \cdot 10^{-4}$ & 0.272 & $8.8 \cdot 10^{-5}$ & 0.330 & $4.5 \cdot 10^{-5}$ & 0.365 & $3.3 \cdot 10^{-5}$ & 0.391 & $2.5 \cdot 10^{-5}$ & 0.411 & $2.1 \cdot 10^{-5}$ & 0.429 & $1.7 \cdot 10^{-5}$ \\
\hline O1-10 & -5.0 & 0.372 & $6.01 \cdot 10^{-5}$ & 0.390 & $4.9 \cdot 10^{-5}$ & 0.469 & $2.8 \cdot 10^{-5}$ & 0.519 & $1.7 \cdot 10^{-5}$ & 0.556 & $1.0 \cdot 10^{-5}$ & 0.586 & $1.3 \cdot 10^{-5}$ & 0.611 & $0.91 \cdot 10^{-5}$ \\
\hline \multirow[t]{2}{*}{ O1-11 } & -8.8 & 0.348 & $5.2 \cdot 10^{-5}$ & 0.362 & $5.0 \cdot 10^{-5}$ & 0.429 & $2.2 \cdot 10^{-5}$ & 0.472 & $1.9 \cdot 10^{-5}$ & 0.502 & $1.0 \cdot 10^{-5}$ & 0.527 & $1.0 \cdot 10^{-5}$ & 0.545 & $1.0 \cdot 10^{-5}$ \\
\hline & & $\alpha 420$ & $\begin{array}{c}r_{\mathrm{d}} \\
t=420\end{array}$ & $\alpha 480$ & $\begin{array}{c}r_{\mathrm{d}} \\
t=480\end{array}$ & $\alpha 540$ & $\begin{array}{c}r_{\mathrm{d}} \\
t=540\end{array}$ & $\alpha 600$ & $\begin{array}{c}r_{\mathrm{d}} \\
t=600\end{array}$ & $\alpha 900$ & $\begin{array}{c}r_{\mathrm{d}} \\
t=900\end{array}$ & $\begin{array}{c}\alpha 120 \\
0\end{array}$ & $\begin{array}{c}r_{\mathrm{d}} \\
t=1200\end{array}$ & & \\
\hline $\mathrm{O} 2-1$ & -4.8 & - & - & - & - & - & - & - & - & - & - & - & - & & \\
\hline $\mathrm{O} 2-2$ & -10.2 & - & - & - & - & - & - & - & - & - & - & - & - & & \\
\hline $\mathrm{O} 2-3$ & -13.6 & - & - & - & - & - & - & - & - & - & - & - & - & & \\
\hline $\mathrm{O} 2-4$ & -18.8 & - & - & - & - & - & - & - & - & - & - & - & - & & \\
\hline $\mathrm{O} 2-5$ & -10.8 & - & - & - & - & - & - & - & - & - & - & - & - & & \\
\hline $01-6$ & -4.6 & 0.840 & $1.7 \cdot 10^{-5}$ & 0.856 & $1.5 \cdot 10^{-5}$ & 0.869 & $1.2 \cdot 10^{-5}$ & 0.880 & $0.74 \cdot 10^{-5}$ & 0.913 & $3.6 \cdot 10^{-6}$ & 0.931 & $3.1 \cdot 10^{-6}$ & & \\
\hline O1-7 & -9.4 & - & - & - & - & - & - & - & - & - & - & - & - & & \\
\hline
\end{tabular}




\begin{tabular}{|cc|c|c|c|c|c|c|c|c|c|c|c|c|} 
O1-8 & -13.8 & 0.547 & $1.8 \cdot 10^{-5}$ & 0.564 & $1.7 \cdot 10^{-5}$ & 0.578 & $1.5 \cdot 10^{-5}$ & 0.583 & $1.1 \cdot 10^{-5}$ & 0.640 & $9.1 \cdot 10^{-6}$ & 0.675 & $5.9 \cdot 10^{-6}$ \\
O1-9 & -17.5 & 0.444 & $1.4 \cdot 10^{-5}$ & 0.457 & $1.5 \cdot 10^{-5}$ & 0.469 & $1.6 \cdot 10^{-5}$ & 0.479 & $1.4 \cdot 10^{-5}$ & 0.546 & $5.5 \cdot 10^{-6}$ & 0.576 & $4.8 \cdot 10^{-6}$ \\
O1-10 & -5.0 & 0.630 & $1.0 \cdot 10^{-5}$ & 0.648 & $0.78 \cdot 10^{-5}$ & 0.664 & $0.64 \cdot 10^{-5}$ & 0.678 & $0.66 \cdot 10^{-5}$ & 0.726 & $3.8 \cdot 10^{-6}$ & 0.762 & $1.9 \cdot 10^{-6}$
\end{tabular}

$\begin{array}{llll}\text { O1-11 } & -8.8 & 0.563 & 0.51 \cdot 10^{-5}\end{array}$

$-\quad-$

a

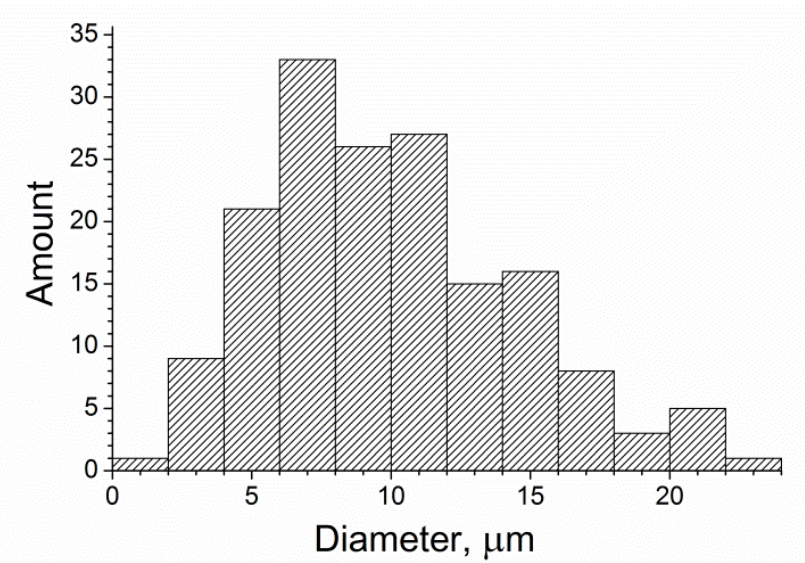

b

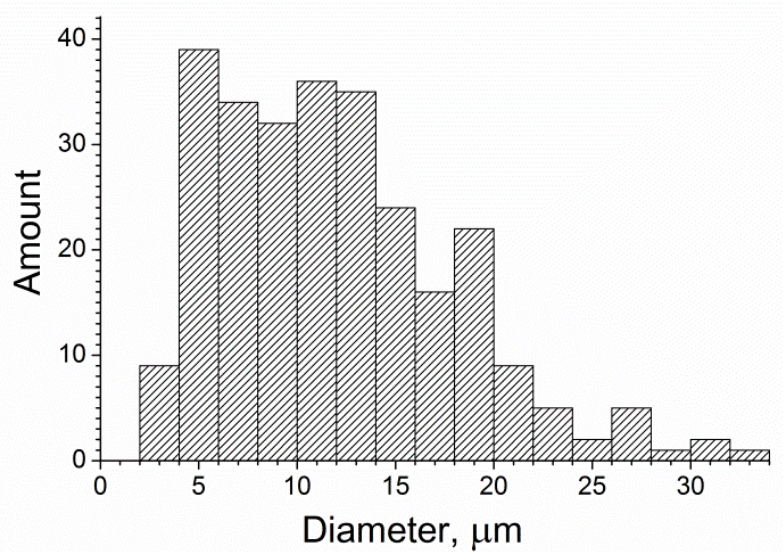

Figure S1. (a) Water droplets size distribution in water-in-oil O2 emulsions (average diameter is $10 \pm 4 \mu \mathrm{m}$ ). (b) Water droplets size distribution in water-in-oil $\mathrm{O} 1$ (average diameter is $12 \pm 6 \mu \mathrm{m}$ ). 